\title{
INFLUÊNCIA DA ADIÇÃO DE LECITINA DE SOJA E DO POLIGLICEROL POLIRICINOLATO NAS PROPRIEDADES REOLÓGICAS DO CHOCOLATE
}

\author{
KARINE MARCONDES DA CUNHA* \\ LEDABATTESTIN QUAST** \\ VALDECIR LUCCAS ${ }^{* \star *}$
}

O objetivo deste trabalho foi avaliar o comportamento reológico de chocolate do tipo meio-amargo, variando-se os teores de lecitina e poliglicerol poliricinolato (PGPR). As amostras de chocolate foram preparadas adicionando-se de 0,3 a 1,4\% $(\mathrm{m} / \mathrm{m})$ de lecitina e a combinação de lecitina/PGPR com PGPR constante de $0,2 \%$ e variando-se o teor de lecitina em 0,3, 0,5 e 0,8\%. As análises reológicas foram realizadas nas temperaturas de $40^{\circ} \mathrm{C}$ (início da temperagem) e de $31^{\circ} \mathrm{C}$ (final da temperagem). No início da temperagem, até o percentual de 0,5\% de lecitina houve redução na viscosidade plástica e aparente. O limite de escoamento apresentou aumento após a adição de 0,5\% de lecitina. Para as amostras contendo lecitina e PGPR, o comportamento das curvas de viscosidade e o limite de escoamento foi semelhante ao das amostras contendo apenas a lecitina. A adição de diferentes teores de emulsificantes não interferiu nos parâmetros da temperagem.

PALAVRAS-CHAVE: CHOCOLATE; VISCOSIDADE; EMULSIFICANTES; ANÁLISES REOLÓGICAS.

Aluna de Iniciação Científica, Engenharia de Alimentos, Universidade Estadual de Ponta Grossa (UEPG), Ponta Grossa, Paraná, Brasil (e-mail: karinemarc@yahoo.com.br).

** Professora Adjunta, Engenheira de Alimentos, UEPG, Ponta Grossa, Paraná, Brasil (email:Ibattestin@uol.com.br).

*** Pesquisador Científico, Instituto de Tecnologia de Alimentos (ITAL), Campinas, São Paulo, Brasil (email: vluccas@ital.sp.gov.br). 


\section{INTRODUÇÃo}

O chocolate tornou-se muito consumido devido às suas características de sabor, associadas ao bem-estar durante a degustação. O chocolate geralmente contém cerca de 28 a $42 \%$ de manteiga de cacau, partículas de sacarose e sólidos de cacau, baunilha como agente aromatizante e lecitina que funciona como emulsificante, cujo conteúdo pode variar de 0,3 a 0,5\% (JOVANOVIC et al., 1995; MIQUEL et al., 2001). Durante o processamento do chocolate ocorrem apropriadas operações técnicas responsáveis pela harmonização dos ingredientes, visando a obtenção de sistema com atributos reológicos e físico-cristalográficos bem definidos (JOVANOVIC et al., 1995; AFOAKWA et al., 2007).

A manteiga de cacau apresenta comportamento polimórfico, ou seja, pode se solidificar sob diferentes formas cristalinas, dependendo da temperatura e do tempo de cristalização, da agitação e, sobretudo, da taxa de resfriamento (MINIFIE, 1989; LUCCAS, 2001; COHEN et al., 2004). Durante o processamento, o chocolate deve ser pré-cristalizado ou temperado antes das etapas de moldagem ou recobrimento. A temperagem deve induzir a formação de núcleos de cristais do tipo beta (ou V) de maior estabilidade termodinâmica, conferindo ao chocolate propriedades de fusão adequadas e retardando a migração de gordura para a superfície do produto (TALBOT, 1994; DHONSI e STAPLEY, 2005).

Os estados polimórficos dos triacilglicerois apresentam propriedades físicas distintas e estão relacionadas com os diferentes arranjos de empacotamento das cadeias hidrocarbonadas presentes nas moléculas dos triacilglicerois durante a sua cristalização (LUCCAS, 2001). Em razão do polimorfismo da manteiga de cacau, o chocolate precisa ser temperado ou pré-cristalizado antes das etapas de moldagem ou recobrimento. O chocolate bem temperado contém aproximadamente de 3 a $8 \%$ de núcleos de cristais do tipo beta com ponto de fusão próximo à temperatura do corpo humano.

Na produção de chocolates é necessário fornecer ao produto propriedades ótimas de fluidez para posterior transformação. O tamanho máximo das partículas influencía tanto o limite de escoamento como a viscosidade plástica (AFOAKWA et al., 2007). O chocolate fundido apresenta propriedades não Newtonianas que podem ser descritas pelos modelos matemáticos de Bingham, Herschel-Bulkley e pelo modelo de Casson (SOKMEN e GUNES, 2006; NESBENY e ZYZELEWJCZ, 2005; AFOAKWA et al., 2009).

A viscosidade da massa de chocolate está relacionada com o processo de fabricação, tamanho e distribuição das partículas. A viscosidade influencía o sabor e a textura e dessa forma a reologia está relacionada com as características sensoriais do produto. Na elaboração de chocolate tornou-se prática muito comum a adição de agentes emulsificantes, que interferem nas suas propriedades reológicas (NEBESNY e ZYZELEWICZ, 2005; AFOAKWA et al., 2007; AFOAKWA et al., 2009).

O chocolate se comporta como uma fase contínua de lipídios e açúcar, contendo o grupo hidrofílico e o grupo hidrofóbico (portanto, não solúvel). Como a interação entre o grupo hidrofílico e a parte hidrofóbica não ocorre espontaneamente, o agente emulsificante torna-se o responsável por essa interação (SOKMEN e GUNES, 2006; AFOAKWA et al., 2007).

A lecitina desempenha função importante na fabricação de chocolate, pois diminui a viscosidade e o limite de escoamento (BECKETT, 1994; NEBESNY e ZYZELEWICZ, 2005). Em torno de 0,5\% de poliglicerol poliricinolato (PGPR) também pode ser usado como agente emulsificante na fabricação de chocolate. Muitas empresas produtoras de chocolates estão utilizando a combinação lecitina/PGPR, porque a lecitina exerce efeito maior sobre a viscosidade plástica e o PGPR maior influência na redução do limite de escoamento (AFOAKWA, PATERSON e FOWLER, 2007).

O objetivo deste trabalho foi avaliar o comportamento reológico de chocolate do tipo meio amargo, utilizando-se diferentes teores de emulsificantes. 


\section{MATERIAL E MÉTODOS}

\subsection{PREPARAÇÃO DAS AMOSTRAS}

O chocolate do tipo meio amargo foi elaborado em laboratório, utilizando-se a seguinte formulação: açúcar (50\%), líquor de cacau (40\%) e manteiga de cacau (10\%).

Para o preparo da massa de chocolate, os ingredientes secos foram homogeneizados em misturador marca Kitchen Aid (modelo K5SS). Após a homogeneização, a mistura foi transferida para refinador de escala piloto marca Drais, composto de 3 cilindros rotativos acoplados a sistema de circulação de água para evitar o super aquecimento.

A determinação do tamanho das partículas foi realizada de acordo com a metodologia utilizada pelo Centro de Tecnologia de Cereais e Chocolates - Cereal Chocotec, do Instituto de Tecnologia de Alimentos (ITAL, 1998). Para a medição do tamanho máximo das partículas, uma amostra representativa do lote foi dispersa em óleo mineral (na proporção aproximada de 1:1) até adquirir consistência homogênea. Usou-se micrômetro manual (marca Mitutoyo) com escala de $0-250 \mathrm{~mm}$, calibrado antes das medidas serem efetuadas. Os resultados apresentados correspondem à média de 20 determinações.

Após o processo de refino, a massa de chocolate foi submetida ao processo de conchagem seca à temperatura de $65^{\circ} \mathrm{C}$ por duas horas em concha marca Inco. Após esse período, a manteiga de cacau foi adicionada ao restante da massa e procedeu-se a conchagem plástica por mais 18 horas. Acrescentou-se $0,1 \%$ de aroma de baunilha sobre a massa do produto final.

Adicionou-se lecitina à massa de chocolate produzida, cujo percentual final variou de $0,3 \mathrm{a}$ $1,4 \%$. A adição de lecitina foi realizada em reator de vidro encamisado, acoplado a banho Brookfield (modelo TC500), à temperatura de $60^{\circ} \mathrm{C}$ durante 15 minutos e agitação constante de 96 rpm.

\subsection{ANÁLISE DA VISCOSIDADE}

Para a análise da viscosidade utilizou-se Reômetro de cilindros concêntricos, dotado de adaptador para pequenas amostras da marca Brookfield (modelo RV-DVIII), e spindle tipo cilíndrico (S15) com relação dos raios dos cilindros interno e externo de 0,75 . O adaptador foi acoplado ao banho termostático da marca Brookfield.

As medidas da viscosidade plástica e o limite de escoamento foram realizadas de acordo com o programa desenvolvido por Vissoto et al. (1999) e descrito na Tabela 1.

\section{TABELA 1 - PROGRAMA USADO PARA A ANÁLISE DA VISCOSIDADE PLÁSTICA E LIMITE DE ESCOAMENTO}

\begin{tabular}{cc}
\hline Tempo & Rotação (rpm) \\
\hline 3 min & 5 \\
3 min & 50 \\
$3 \mathrm{~s}$ & 100 \\
$6 \mathrm{~s}$ & 50 \\
$15 \mathrm{~s}$ & 20 \\
$30 \mathrm{~s}$ & 10 \\
$60 \mathrm{~s}$ & 5 \\
$30 \mathrm{~s}$ & 10 \\
$15 \mathrm{~s}$ & 20 \\
$6 \mathrm{~s}$ & 50 \\
$3 \mathrm{~s}$ & 100 \\
\hline
\end{tabular}


A viscosidade aparente foi medida no mesmo equipamento, utilizando-se rotação de $20 \mathrm{rpm}$ por 30 segundos. As análises de viscosidade plástica e viscosidade aparente foram primeiramente realizadas a $40^{\circ} \mathrm{C}$ (temperatura inicial do processo de pré-cristalização ou temperagem do chocolate) quando ocorre a fusão de todas as formas cristalinas presentes na amostra.

\subsection{TEMPERAGEM OU PRÉ-CRISTALIZAÇÃO}

Com o intuito de verificar a influência da lecitina no processo de pré-cristalização do chocolate foram selecionadas amostras contendo $0,3 \%, 0,5 \%$ e $0,8 \%$ de lecitina. A temperagem dessas amostras foi realizada em temperadeira de laboratório, marca Sollich, conforme metodologia descrita por Talbot (1994).

A temperagem foi efetuada em sala climatizada com temperatura ambiente próxima de $19^{\circ} \mathrm{C}$. Nesse processo, a massa de chocolate permaneceu por 10 minutos na temperatura de $40^{\circ} \mathrm{C}$ para que todos os cristais fossem fundidos. Em seguida, resfriou-se a massa com taxa próxima de $2^{\circ} \mathrm{C} / \mathrm{min}$ até atingir a temperatura de cristalização de $28^{\circ} \mathrm{C}$. A massa de chocolate permaneceu por 8 minutos nessa temperatura, quando efetuou-se o reaquecimento. A temperatura foi elevada e mantida em $31^{\circ} \mathrm{C}$ durante 3 minutos. Para verificar se o processo de pré-cristalização foi adequado mediu-se o índice de temperagem das amostras em equipamento Temperindex, marca Sollich. O instrumento apresenta pequeno porta amostras que é imerso em água refrigerada conectada em leitor de dados que marca a variação da temperatura em determinado intervalo de tempo. A inflexão da curva de resfriamento da amostra dependerá da temperagem realizada (MINIFIE, 1989). De acordo com o manual do equipamento, a pré-cristalização ideal (chocolate temperado adequadamente) apresenta grau de têmpera ou temperindex igual a 5, conforme ilustrado na Figura 1.

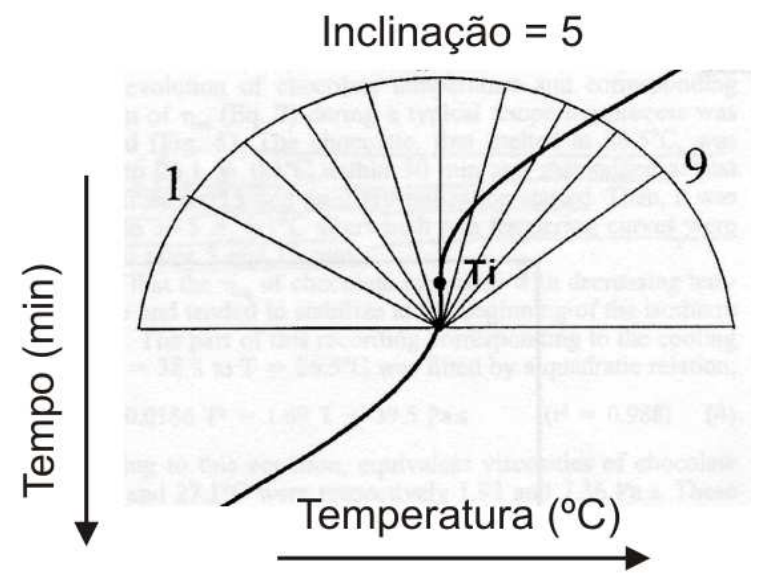

FIGURA 1- DIAGRAMA ESQUEMÁTICO DE CURVA DE RESFRIAMENTO OBTIDA PELO TEMPERÍMETRO QUE CORRESPONDE AO CHOCOLATE ADEQUADAMENTE TEMPERADO

Fonte: LOISEL et al., 1997.

Ao final do processo de temperagem foram realizadas as análises de viscosidade plástica e limite de escoamento, utilizando-se o programa descrito na Tabela 1, porém na temperatura de $31^{\circ} \mathrm{C}$ (temperatura final no processo de pré-cristalização).

Para a verificação da influência do PGPR nos parâmetros reológicos, três amostras com 0,3; 0,5 e $0,8 \%$ de lecitina foram adicionadas de $0,2 \%$ de PGPR.

Nas amostras com lecitina e PGPR foram realizadas as análises de viscosidade plástica e limite de escoamento a $40^{\circ} \mathrm{C}$, utilizando-se as condições já descritas anteriormente. Essas amostras foram temperadas nas mesmas condições das amostras contendo apenas lecitina, medindo-se após o processo de temperagem a viscosidade plástica e o limite de escoamento. 


\section{RESULTADOS E DISCUSSÃO}

\subsection{TAMANHO MÁXIMO DAS PARTÍCULAS}

Após o processo de refino do chocolate, o tamanho médio máximo das partículas foi de $21 \pm 0,008 \mu \mathrm{m}$, semelhante aos valores obtidos por Sokmen e Gunes (2006). Nos processos de fabricação do chocolate é recomendável que a proporção de partículas maiores de $22 \mu \mathrm{m}$ não ultrapassem $20 \%$ do total. O tamanho máximo das partículas é um dos fatores que determinam as propriedades reológicas, pois exerce influência direta nas características sensoriais do produto. Os chocolates que apresentam partículas maiores que $35 \mu \mathrm{m}$ evidenciam característica arenosa (SOKMEN e GUNES, 2006; AFOAKWA, PATERSON e FOWLER, 2007). Por outro lado, partículas menores que $20 \mu \mathrm{m}$ podem elevar a viscosidade do chocolate pela ampliação da área de contato das partículas, aumentando a tensão superficial das partículas. Portanto, partículas muito grandes alteram as características sensoriais do produto final e partículas muito pequenas modificam as características físicas do produto. Nas amostras avaliadas, o tamanho máximo de partículas enquadrou-se na faixa desejada.

\subsection{ANÁLISES REOLÓGICAS DO CHOCOLATE COM LECITINAA $40^{\circ} \mathrm{C}$ (INÍCIO DA TEMPERAGEM)}

A viscosidade aparente não é uma medida constante, pois pode crescer ou decrescer dependendo da taxa de deformação, enquanto a viscosidade plástica é medida pela equação de Casson.

A Figura 2 apresenta a variação da viscosidade plástica e da viscosidade aparente das amostras de chocolate com diferentes teores de lecitina na temperatura de $40^{\circ} \mathrm{C}$.

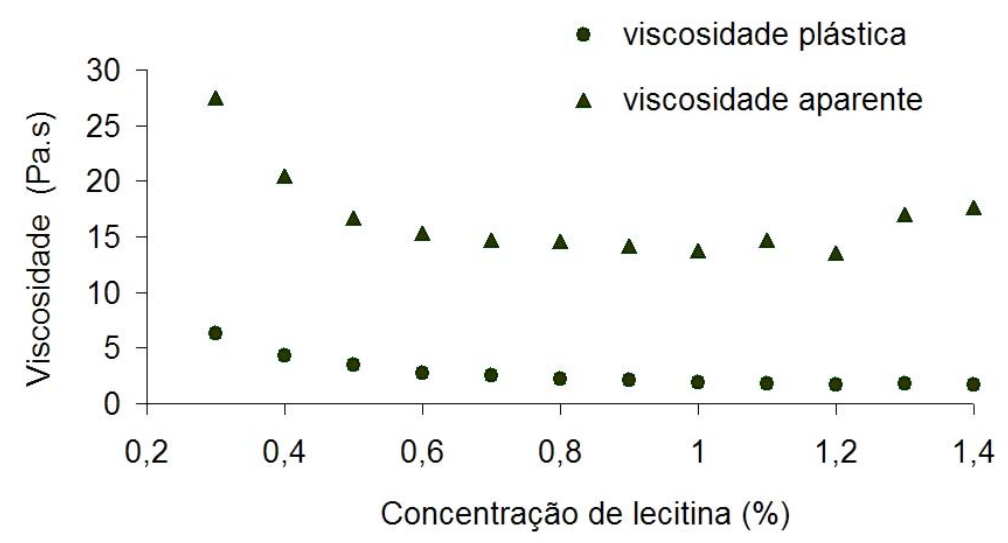

\section{FIGURA 2 - VARIAÇÃO DAS VISCOSIDADES PLÁSTICA E APARENTE NO INÍCIO DA TEMPERAGEM DAS AMOSTRAS COM DIFERENTES TEORES DE LECITINA}

A diminuição mais pronunciada da viscosidade plástica foi observada até a concentração de $0,6 \%$ de lecitina, mantendo-se constante acima desse valor em cerca de 2,5Pa.s. O formato da curva da viscosidade aparente foi similar ao da curva da viscosidade plástica até o valor de 1,2\%, observandose aumento atípico nos valores da viscosidade aparente.

Segundo Vissoto et al. (1997), a lecitina contém entre 65 e 70\% de fosfolipídeos, substâncias tensoativas responsáveis pela formação de filme monomolecular na superfície de partículas como açúcar e cacau, facilitando sua mistura com a gordura. Segundo Nebesny e Zizelewicz (2005), o fragmento hidrofílico da molécula de lecitina associa-se ao açúcar e a parte lipofílica penetra na fase lipídica, reduzindo a fricção interna da massa de chocolate e sua viscosidade plástica.

Segundo Afoakwa et al. (2008), o excesso de lecitina influencía o tamanho dos cristais e o derretimento característico do produto. Com o aumento do teor de lecitina há elevação da viscosidade em 
função do aumento do tamanho dos cristais formados. Os cristais maiores encontram dificuldade de locomoção na massa de chocolate, pois há maior resistência no deslocamento devido à força de atrito existente.

A Figura 3 apresenta a variação do limite de escoamento nas amostras com diferentes teores de lecitina a $40^{\circ} \mathrm{C}$.

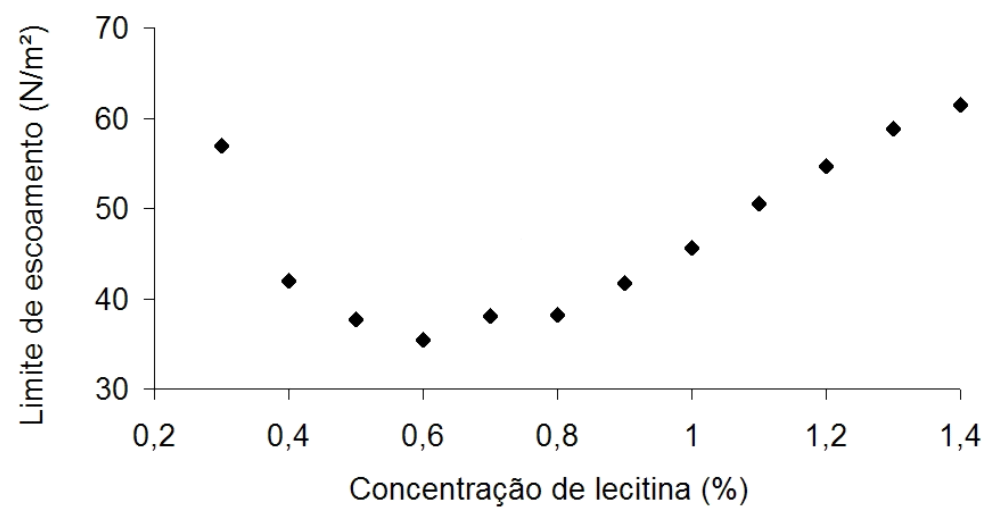

\section{FIGURA 3 - LIMITE DE ESCOAMENTO PARA AMOSTRAS COM DIFERENTES TEORES DE LECITINA NO INÍCIO DA TEMPERAGEM}

O limite de escoamento corresponde à força mínima necessária para que o chocolate comece a escoar. Nas amostras com concentração de $0,6 \%$ de lecitina verificou-se diminuição no limite de escoamento. A partir de $0,7 \%$ de lecitina ocorreu inversão na curva do limite de escoamento. Quando a lecitina é adicionada em excesso no chocolate, sua parte hidrofílica ficará em excesso, enquanto toda a parte hidrofóbica reagirá com a fase lipídica do chocolate. O excesso promove o aumento da viscosidade do chocolate, tornando importante o ajuste da concentração de lecitina para cada tipo de chocolate (HOSKIN e DIMICK, 1980). Pode-se notar que concentrações acima de $0,6 \%$ de lecitina promoveram aumento no limite de escoamento (Figura 3). Observando os resultados da variação da viscosidade (Figura 2) e do limite de escoamento (Figura 3) pode-se recomendar a adição de lecitina até o percentual de $0,6 \%$ para chocolates do tipo meio amargo. A lecitina adicionada em excesso, além de não promover decréscimo na viscosidade, proporciona aumento no limite de escoamento. Sob o ponto de vista econômico, quantidades acima de $0,6 \%$ de lecitina gerariam elevação desnecessária dos custos.

\subsection{AVALIAÇÃO DOS DIFERENTES TEORES DE LECITINA NO PROCESSO DE TEMPERAGEM}

Amostras de chocolate com 0,3; 0,5 e 0,8\% de lecitina foram submetidas ao processo da temperagem. O percentual de $0,3 \%$ foi selecionado por ser a quantidade tradicionalmente utilizada pelas indústrias na fabricação de chocolates. A concentração de $0,5 \%$ de lecitina foi escolhida por tratar-se de valor intermediário e a concentração de $0,8 \%$ de lecitina por pertencer a região da curva em que os valores da viscosidade encontram-se praticamente constantes.

A Tabela 2 apresenta os resultados obtidos para o índice de temperagem nos diferentes ensaios. Os valores apresentados são a média de três bateladas de temperagem avaliadas pelo teste de Tukey.

\subsection{ANÁLISES REOLÓGICAS DO CHOCOLATE COM LECITINAA 31ํ (FINAL DA EMPERAGEM)}

A medida da viscosidade no final da temperagem constitui parâmetro importante, pois nesse ponto o chocolate precisa ser moldado ou utilizado como cobertura para bombons e produtos similares. A Figura 4 mostra o comparativo dos valores da viscosidade plástica obtidos nas temperaturas de $31^{\circ} \mathrm{C}$ (final da temperagem) e $40^{\circ} \mathrm{C}$ (início da temperagem). 


\begin{tabular}{cc}
\hline Concentração de lecitina (\%) & Temperindex \\
\hline 0,3 & $4,97 \pm 1,34^{\mathrm{a}}$ \\
0,5 & $5,10 \pm 0,14^{\mathrm{a}}$ \\
0,8 & $5,35 \pm 0,77^{\mathrm{a}}$ \\
\hline
\end{tabular}

Letras iguais indicam que não houve diferenças estatisticamente significativas ao nível de 95\% de confiança para o índice de temperagem entre as amostras com os diferentes teores de lecitina.

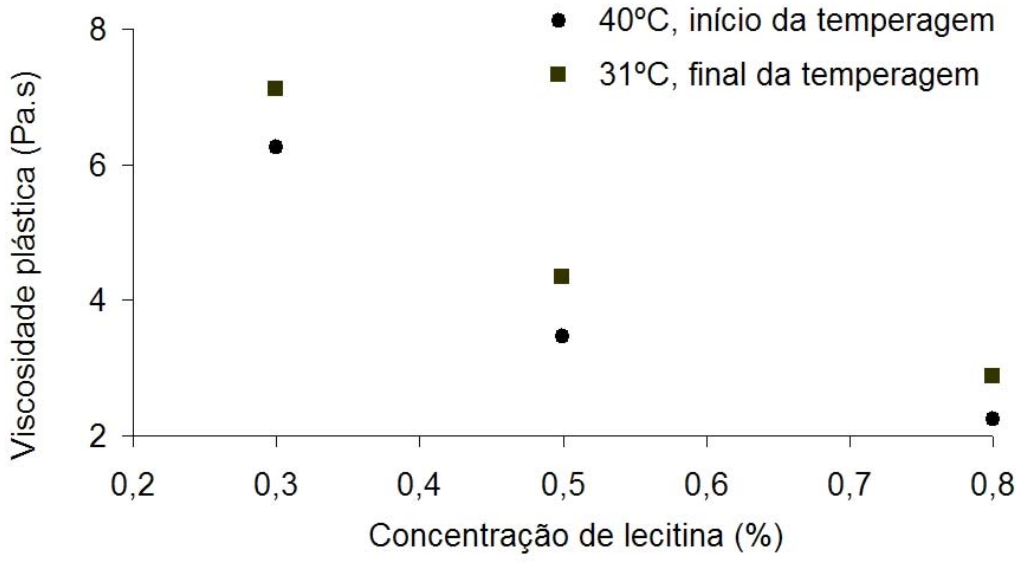

\section{FIGURA 4 - COMPARATIVO ENTRE OS VALORES DA VISCOSIDADE PLÁSTICA NO INÍCIO E AO FINAL DA TEMPERAGEM}

Conforme a Figura 4, tanto no início quanto no final da temperagem, houve decréscimo no valor da viscosidade plástica. $\mathrm{Na}$ temperatura de $31^{\circ} \mathrm{C}$, todos os valores de viscosidade plástica foram superiores aos obtidos a $40^{\circ} \mathrm{C}$. Isso pode ser explicado pelo fato de que no final do processo de temperagem há a presença de núcleos estáveis de cristais do tipo beta que aumentam a viscosidade do chocolate. Adicionalmente, com a diminuição da temperatura ocorre aumento na viscosidade plástica.

A Figura 5 apresenta a variação do limite de escoamento das amostras com 0,3\%; 0,5\% e $0,8 \%$ de lecitina para o início e final da temperagem.

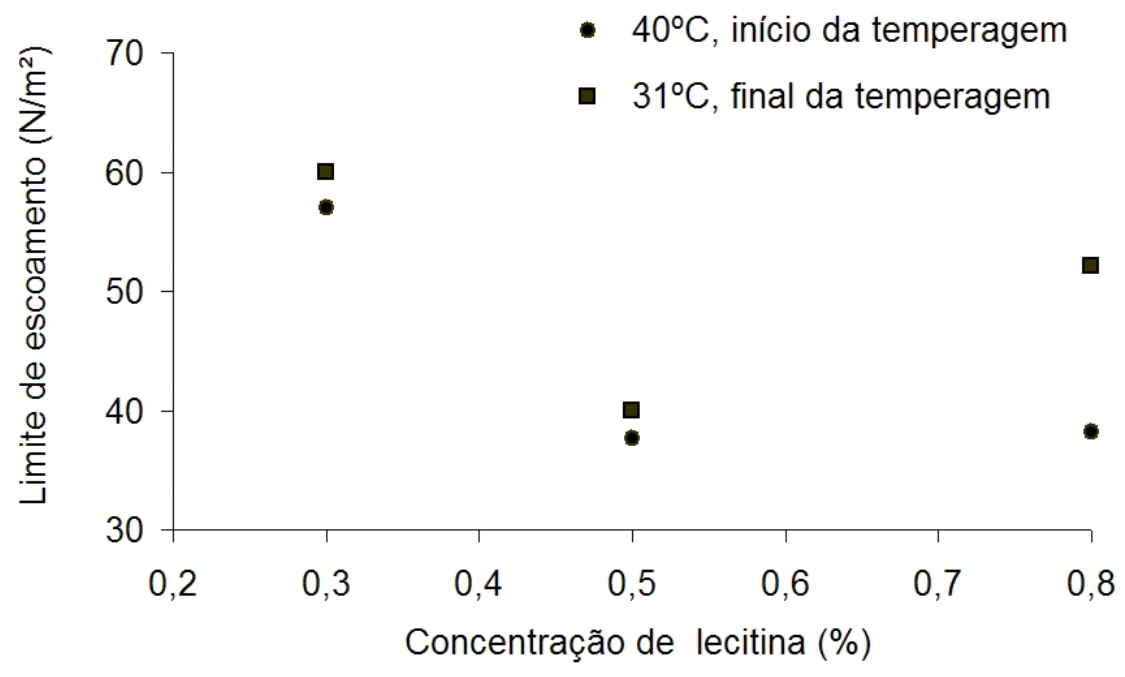

\section{FIGURA 5 - COMPARATIVO ENTRE OS VALORES DO LIMITE DE ESCOAMENTO NO INÍCIO E AO FINAL DA TEMPERAGEM}


O limite de escoamento (Figura 5) mostrou comportamento similar das amostras em ambas as temperaturas até a concentração de $0,5 \%$ de lecitina. À partir dessa concentração, o limite de escoamento a $31^{\circ} \mathrm{C}$ foi maior que a $40^{\circ} \mathrm{C}$, possivelmente, pelo aumento de núcleos de cristais presentes no chocolate e diminuição da interação do chocolate com a lecitina.

\subsection{AVALIAÇÃO DOS DIFERENTES TEORES DE LECITINA E PGPR NO PROCESSO DE TEMPERAGEM}

$\mathrm{Na}$ Tabela 3 estão apresentados os valores obtidos de temperindex ao final da temperagem do chocolate contendo lecitina e PGPR (fixo em 0,2\%). Por meio do teste de Tukey, letras iguais indicam que não houve diferenças estatisticamente significativas ao nível de $95 \%$ de confiança para o índice de temperagem entre as amostras com os diferentes teores de emulsificantes.

\section{TABELA 3 - ÍNDICE DE TEMPERAGEM DO CHOCOLATE CONTENDO LECITINA E PGPR}

\begin{tabular}{ll}
\hline Concentração de emulsificantes & Temperindex \\
\hline $0,3 \%$ lecitina+0,2\% PGPR & $4,20 \pm 0,56^{\mathrm{a}}$ \\
$0,5 \%$ lecitina+0,2\% PGPR & $4,60 \pm 1,80^{\mathrm{a}}$ \\
$0,8 \%$ lecitina+0,2\% PGPR & $5,25 \pm 0,91^{\mathrm{a}}$ \\
\hline
\end{tabular}

Os valores do temperindex encontram-se próximos do valor ideal para chocolates adequadamente temperados (Tabela 3). Pode-se observar que a adição do PGPR não interferiu nos parâmetros de temperagem do chocolate.

\subsection{ANÁLISES REOLÓGICAS DO CHOCOLATE COM LECITINA E PGPR A 31 (FINAL DA TEMPERAGEM)}

Foi verificada a interação dos dois tipos de emulsificantes, lecitina e PGPR, na temperatura de $31^{\circ} \mathrm{C}$ em relação às medidas da viscosidade plástica e do limite de escoamento.

As Figuras 6 e 7 apresentam a viscosidade plástica e o limite de escoamento para as amostras com 0,3; 0,5 e $0,8 \%$ de lecitina e com 0,3; 0,5 e $0,8 \%$ de lecitina acrescidas de $0,2 \%$ de PGPR na temperatura ao final da temperagem $\left(31^{\circ} \mathrm{C}\right)$.

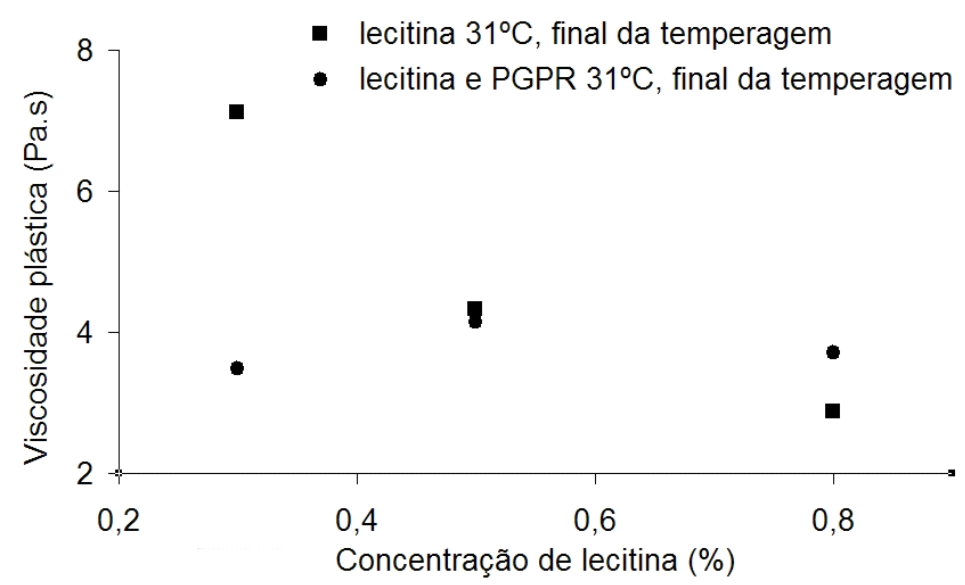

FIGURA 6 - VISCOSIDADE PLÁSTICA DO CHOCOLATE CONTENDO APENAS LECITINA E CHOCOLATE CONTENDO LECITINA E PGPR AO FINAL DA TEMPERAGEM 
A curva referente ao chocolate contendo apenas lecitina apresentou decréscimo na viscosidade, enquanto que a curva referente às amostras com lecitina e PGPR mostrou comportamento praticamente constante. Todas as amostras com lecitina e PGPR apresentaram viscosidade plástica semelhante à da amostra contendo apenas $0,5 \%$ de lecitina. Nas amostras contendo lecitina e PGPR houve redução do limite de escoamento.

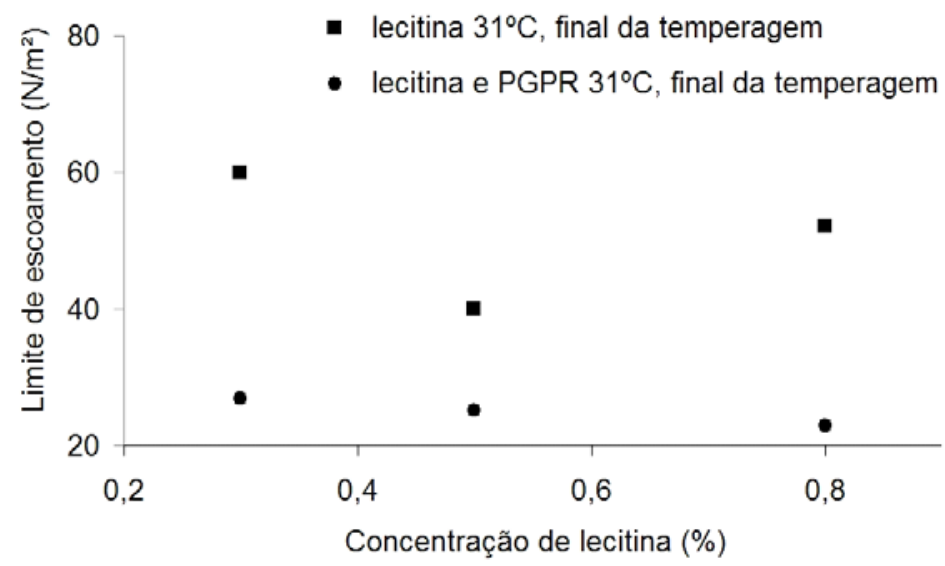

\section{FIGURA 7 - COMPARAÇÃO DO LIMITE DE ESCOAMENTO DO CHOCOLATE CONTENDO APENAS LECITINA COM CHOCOLATE CONTENDO LECITINA E PGPR AO FINAL DA TEMPERAGEM}

Segundo Schantz e Rohm (2005), o efeito da redução do limite de escoamento é significante apenas em frações de $0,4 \%$ de PGPR. A composição das misturas de lecitina e PGPR proporcionará ao chocolate melhores características de escoamento, embora dependa das condições de processamento. De acordo com Beckett (1994), o PGPR tem a particularidade de reduzir fortemente ou até anular o limite de escoamento do chocolate.

\section{CONCLUSÃO}

As amostras contendo até $0,6 \%$ de lecitina evidenciaram redução dos valores de viscosidade plástica e aparente na temperatura de $40^{\circ} \mathrm{C}$. Acima dessa concentração, a viscosidade das amostras permanece praticamente constante, mas ocorre elevação nos valores do limite de escoamento.

A adição de lecitina em 0,3, 0,5 e 0,8\% e o acréscimo de 0,2\% de PGPR não interferiu nas características do processo de temperagem do chocolate tipo meio amargo.

As amostras de chocolate temperado $\left(31^{\circ} \mathrm{C}\right)$ apresentam maiores valores de viscosidade e limite de escoamento quando comparados às amostras na temperatura de $40^{\circ} \mathrm{C}$ (início da temperagem).

A combinação do PGPR com a lecitina proporcionou redução no limite de escoamento quando comparada às amostras contendo apenas lecitina. Com base nos resultados obtidos pode-se sugerir o uso de $0,3 \%$ de lecitina com $0,2 \%$ de PGPR na fabricação do chocolate do tipo meio amargo.

\section{ABSTRACT}

\section{INFLUENCE OF THE ADDITION OF SOY LECITHIN AND POLYRICINOLEATE POLYGLYCEROL ON CHOCOLATE RHEOLOGY}

The objective of this study was to evaluate the rheological behavior of dark chocolates varying lecithin and polyricinoleate polyglycerol (PGPR) levels. Samples of chocolate were prepared adding 0.3 to $1.4 \%$ (w/w) lecithin and a combination of lecithin/PGPR. In these last experiments PGPR was kept constant at $0.2 \%$ and lecithin varied from $0.3,0.5$ and $0.8 \%$. Rheometry analyses were performed at $40^{\circ} \mathrm{C}$ (start of tempering) and $31^{\circ} \mathrm{C}$ (end of tempering). At the start of tempering until $0.5 \%$ lecithin there was a reduction in plastic and apparent viscosity. The flow bound was increased with the addition of $0.5 \%$ lecithin. For samples containing 
lecithin and PGPR, the viscosity curves behavior and the flow bound was similar to the samples containing only lecithin. The addition of different levels of emulsifiers did not interfere in the parameters of tempering.

KEY-WORDS: CHOCOLATE; VISCOSITY; EMULSIFIER; RHEOLOGY DETERMINATIONS.

\section{REFERÊNCIAS}

1 AFOAKWA, E.O.; PATERSON, A.; FOWLER, M.; VIEIRA. J. Comparison of rheological models for determining dark chocolate viscosity. International Journal of Food Science and Technology, London, v.44, n.1, p. 162-167, 2009.

2 AFOAKWA, E.O.; PATERSON, A.; FOWLER, M.; VIEIRA. J. Characterization of melting properties in dark chocolates from varying particle size distribution and composition using differential scanning calorimetry. Food Research International, Oxford, v.41, p.751-757, 2008.

3 AFOAKWA, E.O.; PATERSON, A.; FOWLER, M. Effects of particle size distribution and composition on rheological properties of dark chocolate. Euro Food Research Technology, Berlin, v.226, n.6, p.751-757, 2007.

4 BECKETT, S.T. Industrial chocolate manufacture and use. $2^{\text {nd }}$ ed. London: Chapman and Hall,1994. 408 p.

5 COHEN, K.O.; LUCCAS, V.; JACKIX, M.N.H. Revisão: temperagem ou pré cristalização do chocolate. Brazilian Journal of Food Technology, Campinas, v.7, n.1, p.23-30, 2004.

6 DHONSI, D.; STAPLEY, A.G.F. The effect of shear rate, temperature, sugar and emulsifier on the tempering of cocoa butter. Journal of Food Engineering, Amsterdan, v.77, n.4, p.936-942, 2005.

7 HOSKIN, J.M.; DIMICK, P.S. Observations of chocolate during conching by scanning eletron microcopy and viscosimetry. Journal of Food Science, Chicago, v.45, p.1541-1545, 1980.

8 ITAL. Instituto de Tecnologia de Alimentos. Desenvolvimento de recheios para bombons: ingredientes e parâmetros de controle. Campinas, 1998.

9 JOVANOVIC, O.; KARLOVIC, D.J.; JAKOVLJEVIC, J. Chocolate pre-crystallization: a review. Acta Alimentaria, Budapest, v.24, n.3, p.225-239, 1995.

10 LOISEL, C.; KELLER, G.; LECQ, G.; LAUNAY, B.; OLLIVON, M. Tempering of chocolate in a scraped surface heat exchanger. Journal of Food Science, Chicago, v.62, n.4, p.773-780, 1997.

11 LUCCAS, V. Fracionamento térmico e obtenção de gorduras de cupuaçu alternativas à manteiga de cacau para uso na fabricação de chocolate. 2001. 195 p. Tese (Doutorado em Engenharia Química) - Faculdade de Engenharia Química, Universidade Estadual de Campinas, Campinas.

12 MINIFIE, B.W. Chocolate, cocoa and confectionary: science and technology. $3^{\text {rd }}$ ed. New York: Chapman \& Hall 1989. 904 p.

13 MIQUEL, M.E.; CARLI, S.; COUZENS, P.J.; WILLE, H.J.; HALL, L.D. Kinetics of the migration of lipids in composite chocolate measured by magnetic resonance imaging. Food Research International, Oxford, v.34, p.773-781, 2001.

14 NEBESNY, E.; ZYZELEWICZ, D. Effect of lecithin concentration on properties of sucrose-free chocolate masses sweetened with isomalt. Euro Food Research Technology, Munchen, n.220, p.131-135, 2005.

$15 \mathrm{SCHANTZ,} \mathrm{B.;} \mathrm{ROHM,} \mathrm{H.} \mathrm{Influence} \mathrm{of} \mathrm{lecithin-PGPR} \mathrm{blends} \mathrm{on} \mathrm{the} \mathrm{rheological} \mathrm{properties} \mathrm{of} \mathrm{chocolate.} \mathrm{Lebensmittel}$ Wissenschaf Technologie, n.38, p.41-45, 2005.

16 SOKMEN, A.; GUNES, G. Influence of some bulk sweeteners on rheological properties of chocolate. LWT Food Science and Technology, Amsterdam, v.39, n.10, p.1053-1058,2006.

17 TALBOT, G. Chocolate temper. In: BECKETT, S.T (Ed.). Industrial chocolate manufacture and use. $2^{\text {nd }}$ ed. London: Chapman \& Hall, 1994. 408 p.

18 VISSOTO, F.Z.; LUCCAS, V.; BRAGAGNOLO, N.; TURATTI, J.M.; GRIMALDI, R.; FIGUEIREDO, M.S. Características físico-química e reológica de chocolates tipo cobertura elaborado com gorduras alternativas. Brazilian Journal of Food Technology, Campinas, n.2, p. 139-148, 1999.

19 VISSOTO, F.Z.; SOLER, M.P.; VITALI, A.A.; LUCCAS, V.; BRAGAGNOLO, N.; TURATTI, J.M.; SZUSTER,R. Propriedades reológicas de chocolates comerciais tipo cobertura. Coletânea do ITAL, Campinas n.27, p.127-237, 1997. 